\title{
Increasing access to microfluidics for studying fungi and other branched biological structures
}

\author{
Larry J. Millet ${ }^{1,2^{*}}$ (D) Jayde Aufrecht ${ }^{2,3}$, Jessy Labbé ${ }^{1,4}{ }^{\mathbb{D}}$, Jessie Uehling ${ }^{5,6}$, Rytas Vilgalys ${ }^{5}$, Myka L. Estes ${ }^{7}$, \\ Cora Miquel Guennoc ${ }^{1,8}$, Aurélie Deveau ${ }^{8}$, Stefan Olsson ${ }^{9}$, Gregory Bonito ${ }^{10}$, Mitchel J. Doktycz ${ }^{1,2}$ [D \\ and Scott T. Retterer ${ }^{1,2,3}$ (1)
}

\begin{abstract}
Background: Microfluidic systems are well-suited for studying mixed biological communities for improving industrial processes of fermentation, biofuel production, and pharmaceutical production. The results of which have the potential to resolve the underlying mechanisms of growth and transport in these complex branched living systems. Microfluidics provide controlled environments and improved optical access for real-time and high-resolution imaging studies that allow high-content and quantitative analyses. Studying growing branched structures and the dynamics of cellular interactions with both biotic and abiotic cues provides context for molecule production and genetic manipulations. To make progress in this arena, technical and logistical barriers must be overcome to more effectively deploy microfluidics in biological disciplines. A principle technical barrier is the process of assembling, sterilizing, and hydrating the microfluidic system; the lack of the necessary equipment for the preparatory process is a contributing factor to this barrier. To improve access to microfluidic systems, we present the development, characterization, and implementation of a microfluidics assembly and packaging process that builds on self-priming point-of-care principles to achieve "ready-to-use microfluidics."
\end{abstract}

Results: We present results from domestic and international collaborations using novel microfluidic architectures prepared with a unique packaging protocol. We implement this approach by focusing primarily on filamentous fungi; we also demonstrate the utility of this approach for collaborations on plants and neurons. In this work we (1) determine the shelf-life of ready-to-use microfluidics, (2) demonstrate biofilm-like colonization on fungi, (3) describe bacterial motility on fungal hyphae (fungal highway), (4) report material-dependent bacterial-fungal colonization, (5) demonstrate germination of vacuum-sealed Arabidopsis seeds in microfluidics stored for up to 2 weeks, and (6) observe bidirectional cytoplasmic streaming in fungi.

\footnotetext{
*Correspondence: Imillet1@utk.edu

${ }^{2}$ The Bredesen Center, University of Tennessee-Knoxville, Knoxville, TN 37996, USA

Full list of author information is available at the end of the article

This manuscript has been authored by UT-Battelle, LLC under Contract No. DE-AC05-00OR22725 with the U.S. Department of Energy. The United States Government retains and the publisher, by accepting the article for publication, acknowledges that the United States Government retains a non-exclusive, paid-up, irrevocable, worldwide license to publish or reproduce the published form of this manuscript, or allow others to do so, for United States Government purposes. The Department of Energy will provide public access to these results of federally sponsored research in accordance with the DOE Public Access Plan (http://energy.gov/downl oads/doe-public-access-plan).
}

(c) The Author(s) 2019. This article is distributed under the terms of the Creative Commons Attribution 4.0 International License (http://creativecommons.org/licenses/by/4.0/), which permits unrestricted use, distribution, and reproduction in any medium, provided you give appropriate credit to the original author(s) and the source, provide a link to the Creative Commons license, and indicate if changes were made. The Creative Commons Public Domain Dedication waiver (http://creativecommons.org/ publicdomain/zero/1.0/) applies to the data made available in this article, unless otherwise stated. 
Conclusions: This pre-packaging approach provides a simple, one step process to initiate microfluidics in any setting for fungal studies, bacteria-fungal interactions, and other biological inquiries. This process improves access to microfluidics for controlling biological microenvironments, and further enabling visual and quantitative analysis of fungal cultures.

Keywords: Fungi, Microfluidics, Cell culture, Bacterial-fungal interactions, Plant root, Arabidopsis, Neuron, Branching biology

\section{Background}

Morphological branching is a common and fundamental mode of biological propagation and growth [1-4]. Branched biological structures are evident across all taxonomic kingdoms and size scales. Aggregates of unicellular bacteria form branching communities, which gives rise to colonial phenotypes that are often distinct (e.g. morphology and color) [5-10]. From roots to branches, and stems to leaf structures; plants arborize to develop high surface area tissues; a plants branching structure is vital for nutrient absorption in photosynthesis, energy transport and storage, reproduction, and waste release [11]. Hyphal branching in fungi is involved in beneficial and detrimental interactions among plants and microbes, it is through these high surface-area structures that nutrient uptake, environmental signaling, and communication are achieved [12-14]. Neuronal cells and tissues branch to connect with targets cells and organs for efficient computation of information and the coordination of physiological processes $[15,16]$. Understanding the physical and molecular cues that initiate the formation and function of branching structures and resolve the underlying mechanisms of growth and transport in branched tissues will benefit relevant industries including those involved in fermentation, biofuel production, and health care. To capture the dynamics of this process at such a fine spatial scale requires a culturing platform that enables real-time and high-resolution imaging. While microtechnological methods are well established for culturing neurons and mammalian cells, advancements for increasing the precision and sophistication for measuring plant, fungal, and microbial structures and dynamics (e.g. growth, forces, secretions) are in demand [17-21].

Microfluidic platforms are particularly well-suited for guiding, restraining, and imaging branched structural growth and specification; through a modular on-chip approach, branched biological systems can be interrogated in ways not possible via conventional culture. For example, customized microfluidic designs with interconnected compartments may be used to facilitate and isolate desired branching features. Highly-controlled microfluidic environments also improve the ability to study innate mechanisms and environmental influences guiding process outgrowth, cellular physiology, and biological interactions [22-26]. Despite these benefits, the intersection of microfluidics and biology is highly interdisciplinary and often requires strong cross-departmental or multi-institutional collaborations in order to apply the precision technology toward answering deeplyrooted biological questions. Microfluidic applications for rhizosphere-on-a-chip are providing high-resolution access for studying the dynamics of root-bacterial interactions [27-29]. Recent studies on fungal polarity and growth, and plant development and metabolism demonstrate the ability to increase throughput and semi-automate work to resolve molecular regulations of mesoscale biological interactions using microfluidics [30,31].

Despite the numerous papers that have sought to simplify microfluidics use for biological inquiry [32, 33], technical and logistical barriers still exist that counteract the effective deployment of microfluidics in biological disciplines. Technical barriers to bio-microfluidic collaborations include equipment, such as vacuum pumps, house vacuum systems, syringe pumps, fabrication facilities. Physical barriers include institutional proximity. Unfortunately, the lack of familiarity with microfluidic systems (creation and implementation) and personal contacts are also primary impediments to initiating collaborations. Overcoming these limitations will promote the broad use of a microfluidics tool-set for biological inquiry. For example, microfluidic platforms can readily be designed to provide an environment that allows for resolving the biological response to local physical, chemical, and biological cues integrated within the platform design [34-37]. Complex barriers, topographical cues, compartmentalization of chemically and physically interacting biological systems, and complex chemical gradients can be controlled and manipulated within microfluidic systems to replicate natural interactions and conditions in a fundamental form [22, 38-42].

Assembling, sterilizing, and hydrating the microsystem is one of the principle technical barriers that limits the use and implementation of microfluidics in biological investigation. The lack of both simple and precision equipment is a key contributing factor that further complicates the use of microfluidics [43]. In the effort to help collaborators overcome barriers to implementing microfluidics, we have resolved a preparation strategy 
that greatly minimizes the hurdles associated with using microfluidics in biology. Here, we present the development, characterization, and implementation of a microfluidics assembly process that builds on principles employed in self-priming and point-of-care technologies [44-49]. We designed and implemented novel microfluidic chips, and also tested previously implemented plant-chip platforms [29], toward validating the utility of ready-to-use packaging to overcome common implementation barriers for environmental science research and for new users of microfluidics. We believe that this approach is applicable for a variety of different platform architectures and a wide range of biological studies. The result of our fabrication process is a sterile, ready-to-use microfluidic system that can be implemented anywhere. We demonstrate initial results from international collaborations focusing on the biology of filamentous fungi and include other applications for branching samples such as plants and neurons. In these biological systems, microfluidics enable real-time visualization and quantification of fungi, plants and multispecies interactions.

\section{Methods}

Methods for the fabrication and assembly of microfluidics are detailed in Additional file 1. Figure 1 summarizes the fabrication and assembly process used to prepare 'ready-to-use microfluidics.'

\section{Vacuum packaging}

Fully assembled microfluidic channels on glass slides, or in glass-bottom dishes, were first equilibrated under vacuum $(90 \mathrm{kPa}, 30 \mathrm{~min})$ in a sealed glass desiccator. Then, channel assemblies were immediately removed from the vacuum chamber and transferred to thermal-sealable pouches and immediately vacuum-sealed $(82 \mathrm{kPa}$, Food Saver model \#V3240) for transport and until the time of use. To determine the 'best if used by date', 35 microfluidic channel samples were vacuum packed and marked with the test date. To measure the time for the microfluidics to fill, channels were removed from vacuum-sealed pouches and loaded with water containing food color dye; the time from dye addition to channel fill was documented and plotted (Fig. 2).

\section{Microfluidic design and operation}

Spoke-wheel microfluidics This novel chip architecture (new and not previously published) consists of a central circular chamber $(8 \mathrm{~mm})$ with the roof supported by pillars $(n=118$ dia.$=100 \mu \mathrm{m})$. Sixteen primary radiating channels $(l=1.5 \mathrm{~mm}, w=500 \mu \mathrm{m})$ emanate from the central chamber to terminate at a primary concentric channel $(r=6 \mathrm{~mm}, w=500 \mu \mathrm{m})$. Secondary radial channels $(n=123$, dia.$=4 \mathrm{~mm})$ connect the primary and secondary concentric channels. The depth of the entire channel system is different for fungal cultures $(h=11 \mu \mathrm{m})$ and neuronal cultures $(h=50 \mu \mathrm{m})$.

Two ports at the ends of the peripheral channel permit fluidic exchange. The middle 'accessory' port between the center well and the peripheral ports allows for efficient priming of the microfluidic space; for bacterial-fungal interaction studies, this port is for loading agar into the central chamber. For bacterial-fungal interactions, the center well is used for inoculating fungi, the primary concentric channel is used for observing bacterial-fungal interactions, and the peripheral channel is used for inoculating bacteria. For neuronal cultures, the center well is used for the introduction of dissociated neurons, the peripheral channel ports are used for media exchange.

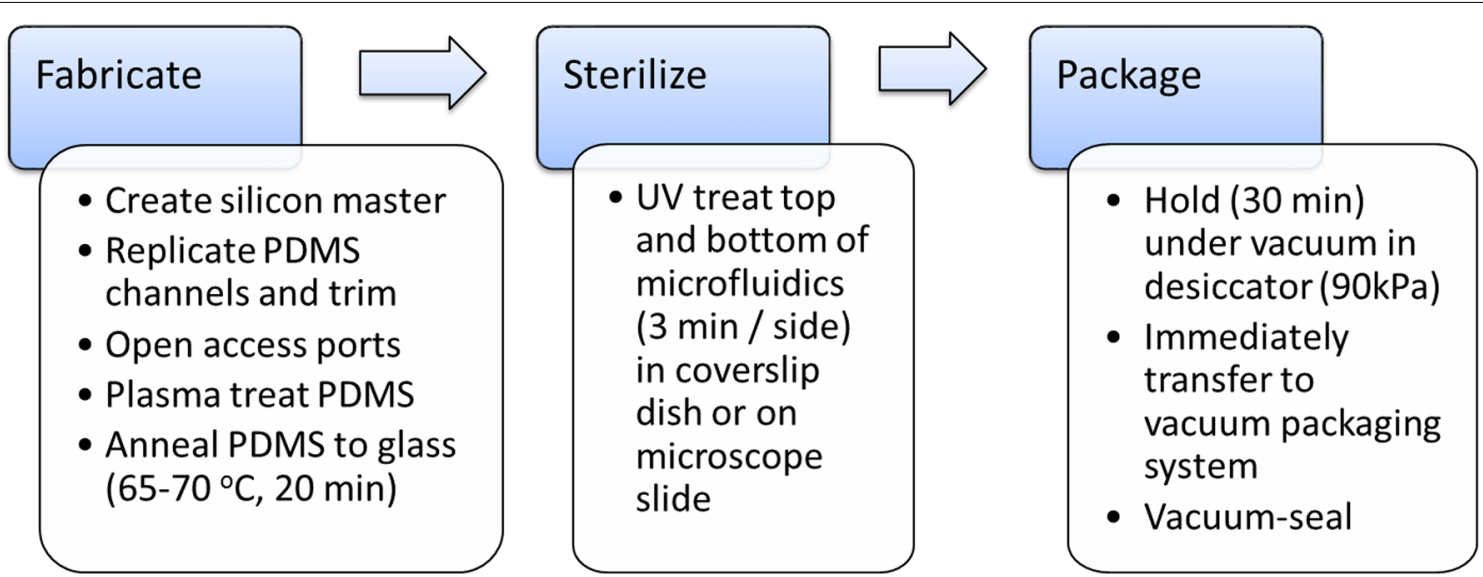

Fig. 1 An overview of the fabrication and assembly protocol for creating ready-to-use microfluidics. Standard photolithography is used to create microfluidic masters, conventional replicate molding with PDMS is used to produce microfluidic structures that are annealed to glass slides or coverslips. UV light exposure or autoclaving processes are used to sterilize the microfluidic platforms. Equilibration under vacuum is the conditioning step that immediately precedes vacuum packaging to produce a ready-to-use microfluidic platform 
a
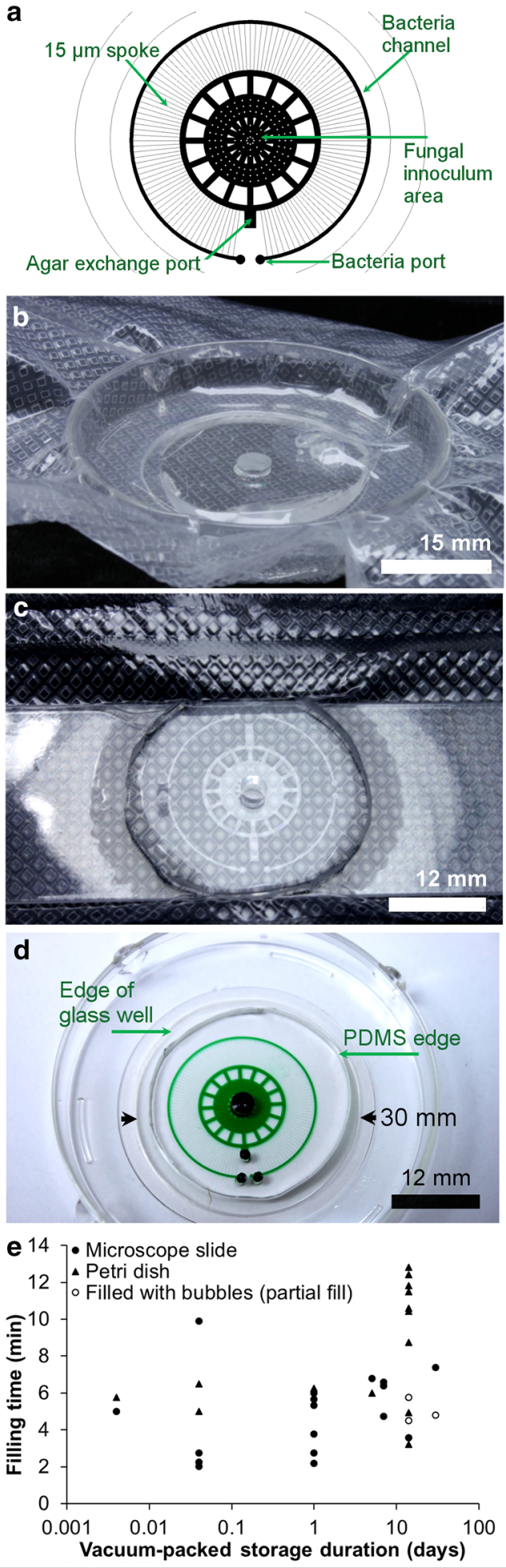

Fig. 2 Sterile pre-packaged ready-to-use microfluidics. a Design of the spoke-wheel device used for characterizing the vacuum-packaging and use process, and for quantifying the 'best if used by date.' $\mathbf{b}$ Spoke wheel microfluidic chamber with integrated PDMS tubing in a glass-bottom dish, vacuum-equilibrated (20 min), then vacuum-sealed for storage, transport, or direct use. c Autoclaved microscope slide with spoke-wheel microfluidic culture chamber in a vacuum-sealed pouch. $\mathbf{d}$ The spoke wheel microfluidic device in a coverslip-bottom dish filled with water containing green food-dye. e Vacuum packaged microfluidics on microscope slides or in coverslip-bottom dishes maintain ability to fill ( $<2$ weeks) within 13 min

"ORNL" chambers This novel architecture (new and not previously published) provides two parallel boundary channels that connect two separate ports. Parallel boundary channels are connected through a grid of microfluidic channels $(w=15 \mu \mathrm{m}, h=17 \mu \mathrm{m})$. Within the grid between the boundary channels are four chambers formed of the letters "ORNL." Pitch of the 15- $\mu \mathrm{m}$ channel grid is $\sim 500 \mu \mathrm{m}$ for the smaller array. This design enables fungal growth in chambers for hyphal isolation and bacterial fungal interaction studies.

Root chip microfluidic This microfluidic architecture was previously designed for Arabidopsis seed germination and growth rate measures, and has been published for root-bacteria interaction studies $[29,50]$. Here, this device is used to demonstrate that prior art, such as the seed-in-chip system, can be incorporated into this fabrication process for decreasing barriers to implementing microfluidics in collaborations. Channel dimensions include a central channel $(4 \mathrm{~mm} l, 200 \mu \mathrm{m} w, 200 \mu \mathrm{m} h)$ guides the primary root shaft down a larger $(20 \mu \mathrm{m}$ tall, $1 \mu \mathrm{L}$ ) rectangular chamber for bathing the growing root hairs. The PDMS microfluidic chip was exposed to air plasma prior to conformal contact and thermal annealing $\left(70{ }^{\circ} \mathrm{C}\right)$. The channel-on-slide structures were cleaned by autoclave sterilization prior to vacuum-packaging of Arabidopsis seeds.

\section{Cell cultures}

Protocols for preparations and cell cultures of eukaryotic and prokaryotic cells (fungi, plant, bacteria, and neuronal cultures) are modified from prior reports and detailed in supplementary information [51-64].

\section{Results}

The fabrication and assembly of PDMS-based microfluidics for fungi, roots, and neurons was achieved through conventional photolithographic processes yielding 
PDMS-channels on microscope slides or coverslip-bottomed dishes. UV-treatment (for glass-bottomed dishes), or autoclave sterilization (microscope slides), produces a sterile microfluidic platform that is amenable for cell culture studies (Fig. 1). Figure 2a-d shows that microfluidics on glass-bottomed dishes (with or without lids) or microscope slides can be vacuum sealed in plastic pouches for shipping, and later, fluidic priming. Low viscosity solutions (e.g. culture media or water) completely fill the entire channel network within minutes after removing the PDMS spoke-wheel microfluidics from the vacuum chamber. Figure 2e shows that vacuum-packaged microfluidics continue to fill with culture media toward completion, if used within 14 days of the vacuum sealing date.

Prior to distributing vacuum-packed microfluidics to collaborators, we characterized the feasibility of our spoke-wheel microfluidic design to permit the observation of bacterial-fungal interactions for high resolution imaging studies. Figure 3a, b shows a spoke-wheel microfluidic architecture that promotes fungal-bacterial interaction studies in microfluidics. The agar-filled central chamber promotes fungal growth while stabilizing the fungal inoculum during culture and shipping (Fig. 3b). The peripheral concentric channel enables the introduction of bacteria at the periphery of the microenvironment. Center to edge, the radiating channels maintain a similar culture space and volume in the channels while providing increasingly narrow passages for the navigation of the fungal hyphae. The central chamber (without the culture well) has a volume of $0.04 \mu \mathrm{L}$, the 16 primary radiating channels (large channels) with the primary concentric channel retains $0.03 \mu \mathrm{L}$, and the sum of the 63 spokes (small channels) is $0.04 \mu \mathrm{L}$. Figure 3c shows a summary of the number of hyphae that colonize the intersection of the spoke-wheel of the platform following 2 weeks in culture. In Fig. 3c, an asterisk and hash symbol in the inset and data bar demarcate the corresponding values for regions of the device quantified as entering and exciting the large microfluidic channel prior to entering the small microfluidic spoke channel. Figure $3 d$, e is an example of hyphae in the large microfluidic channel, on average two hyphae occupy the intersection of the primary concentric circle and the hyphal spoke to permit a low-density of hyphae for resolving bacteria-fungal interactions. As the fungal cultures continue to grow and mature, the fungal hyphae reproducibly fill the device.

After 3 days of co-culturing bacteria in the microfluidic environment, individual hyphae and bacterial colonizations can be observed in the $15 \mu \mathrm{m}$-wide radiating (spoke) channel (Additional file 2: Figure S1). After 4 weeks in culture, fungal hyphae navigate the entire fluidic architecture with bacteria to intersect in open places and align in confined spaces. Probiotic biofilms of the mutualistic Pseudomonas fluorescens GM41 become established on the surface of Laccaria bicolor hyphae [65, 66]. We observed that L. bicolor hyphae show preference for the PDMS surface over the glass surface (Fig. 3f(iii)), which is softer and more gas permeable. The bacterial colonies form at either glass or PDMS interfaces, but colony prevalence is substantially reduced at the glassfluid interface (Fig. 3f(i)) as compared to the PDMS-fluid interface (Fig. 3f(ii)). Through collaboration, high-resolution images of fluorescent $P$. fluorescens BBc6 colonization of fungal hyphae were observed (Fig. 3g).

In minimal media, $P$. fluorescens GM41 cells navigate along the surface of Laccaria bicolor S238N hyphae in vitro prior to colonization on or adjacent to the hyphae (Additional file 3: Figure S2). A standard deviation image of a an image stack ( 25 frames) from a time-lapse image series $(6 \mathrm{~s})$ shows a range of densities of bacteria (white borders on fungi against a black background) moving along Laccaria hyphae (Additional file 3: Figure S2). Kymographs (line traces over time displayed in a twodimension image) clarify the range of densities for which bacterial transport occurs on adjacent segments of the hypha (Additional file 3: Figure S2). But the biological question remains to be answered exactly how bacteria select colonization sites on fungal hyphae. While fungal

\footnotetext{
(See figure on next page.)

Fig. 3 Microfluidics for bacterial-fungal interaction studies. a The spoke-wheel microfluidic design incorporates ports for separate, spatially-defined inoculations of fungi and bacteria. b Shown are green dye-filled channels (loaded with vacuum-assisted filling) in a glass-bottomed dish. An agar plug fills the center of the chamber introduced through the center culture well and aspirated through the agar exchange port. The agar plug holds the fungal inoculum in place and minimizes dehydration during growth. For scale, the open glass surface (between black arrows) is $30 \mathrm{~mm}$. c Average number of hyphae per primary and secondary radiating channels from a single dish (15 DIV). The inset image shows channel locations that correspond to the graphed data. $\mathbf{d}$, e Bacterial-fungal interactions are conveniently established and imaged with microfluidic systems. Image d and inset e of Pseudomonas fluorescens GM41 navigating the Laccaria bicolor fungal highway within a microfluidic chamber (3 DIV bacteria co-culture). $\mathbf{f}(\mathrm{i}, \mathrm{ii})$. fluorescens GM41 bacterial communities accumulate where fungi contact the glass (i) or PDMS (ii) surface, preferentially forming at the PDMS-hyphae surface $\mathbf{d}$ over the glass-hyphae interface (30 DIV co-culture). $\mathbf{g}$ In the same device architecture, Pseudomonas fluorescens BBc6 biofilm-like accumulation on ectomycorrhizal fungi (L. bicolor S238N) 16 h after bacterial inoculation. $\mathbf{h}$ Vacuum-packed spoke-wheel microfluidics are permissive for even the most sensitive cell cultures, neurons. Here, neurons (DIV 4) were transfected for molecular imaging studies (VAMP2, magenta; PSD-95, green)
} 

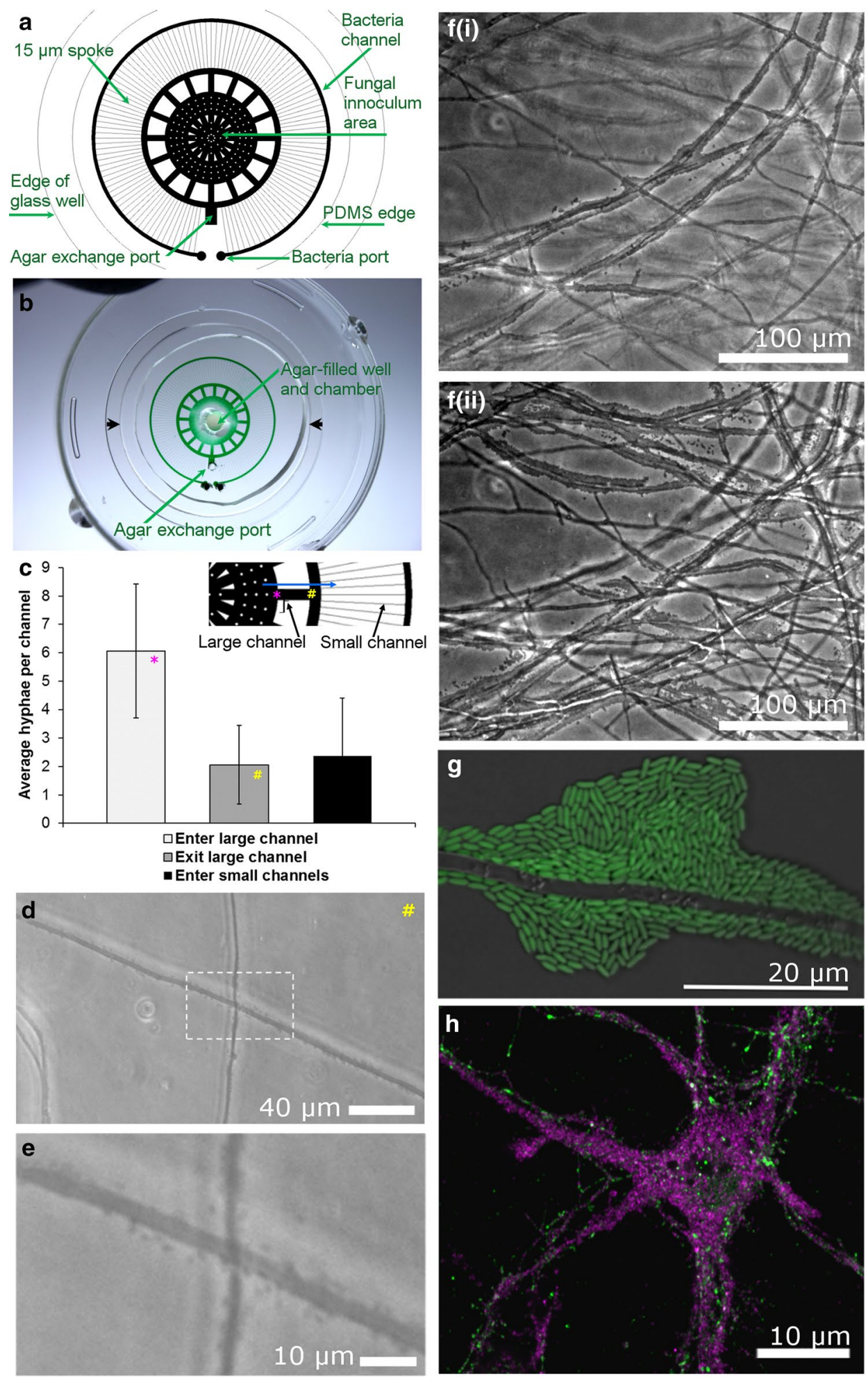

highway and bacterial-fungal interactions can be imaged outside of microfluidics, these microsystems make the process more convenient by preventing drying and enabling confinement over long culture periods to image and map bacterial motility and colonization on hyphal outgrowths. 
Fig. 4 Root-chip microfluidic channel for packaging, storing, culturing, and imaging Arabidopsis roots. a The chip design contains a single root culture channel and accessory ports for sampling and delivery of media and microbes. $\mathbf{b}$ A vacuum-packaged microfluidic root-chip shown with the same orientation. $\mathbf{c}$ Arabidopsis root growing in the central microfluidic channel with marks for measuring growth. $\mathbf{d}$ Growth data for vacuum-packed and control seeds. The packaging process does not attenuate growth conditions of stored roots ( $<14$ days at room temperature)

With this vacuum packaging process being amenable for coverslip-bottomed dishes, with or without lids, we prepared 22 dishes capped with lids in vacuum-sealed pouches. Nineteen dishes were shipped to collaborators for culturing cortical neurons for transfection and molecular imaging of neuronal connections; three dishes were retained in-house. Of the 22 coverslip-bottomed dishes, three coverslips buckled due to the vacuum pressure and pouch pressing on the back of the fragile coverslip window. Placing the glass-bottomed dish within the lid of a $50 \mathrm{~mm}$ Petri dish stabilized the glass and solved the issue of the collapsing cover glass. With vacuumpackaged microfluidics, collaborators that are new to microfluidics were able to achieve neuronal cultures in challenging culture volumes and microfluidic dimensions (Fig. 3h).

Figure $4 \mathrm{a}-\mathrm{d}$ shows seed germination results for Arabidopsis thaliana seeds cultivated in a root-chip system. In prior work, this system supported bacterial-plant interaction studies; here, we vacuum packed $A$. thaliana seeds in the system to determine if the packaging process negatively influenced the seed germination process. After $7(n=3)$ and $14(n=7)$ days of storage in the vacuum packaging (kept in the dark), 100\% of the seeds germinated within 3 days of opening the package and filling the chamber with Murashige-Skoog medium (Fig. 4d). Of these germinated seedlings, all of the roots from the 7-day storage and two of the roots from the 14-day storage test grew into the large microfluidic channel. By the second and third days in vitro, the growth rate of the roots for the vacuum-stored seedlings in the channel were not significantly different from the growth rate of non-vacuumed control $(\mathrm{n}=3)$ seedlings (Fig. $4 \mathrm{~d})$, although initial growth rates at day 1 were significantly different ( $p=0.0227)$, unpaired $t$ test, $p<0.05$.

To improve access to microfluidics for fungal-bacterial interaction studies by microbiologists, we developed a universal chamber-grid architecture amenable for culturing fungi, bacteria, or bacterial-fungal co-cultures.
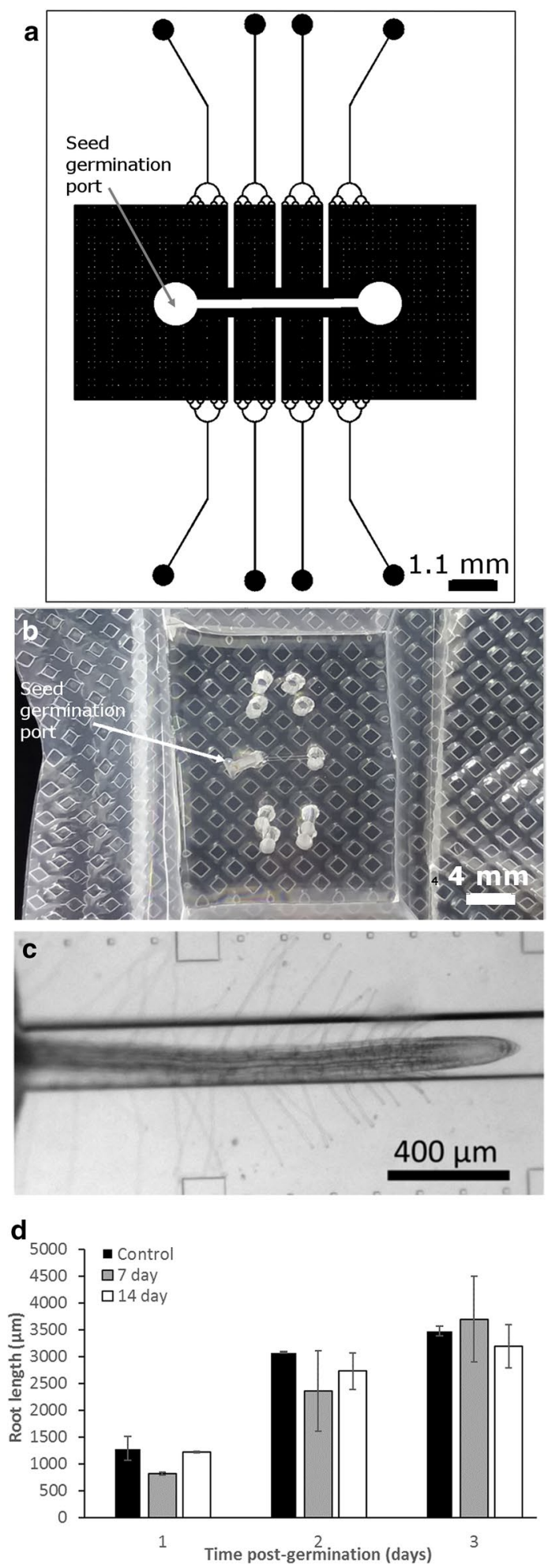
a
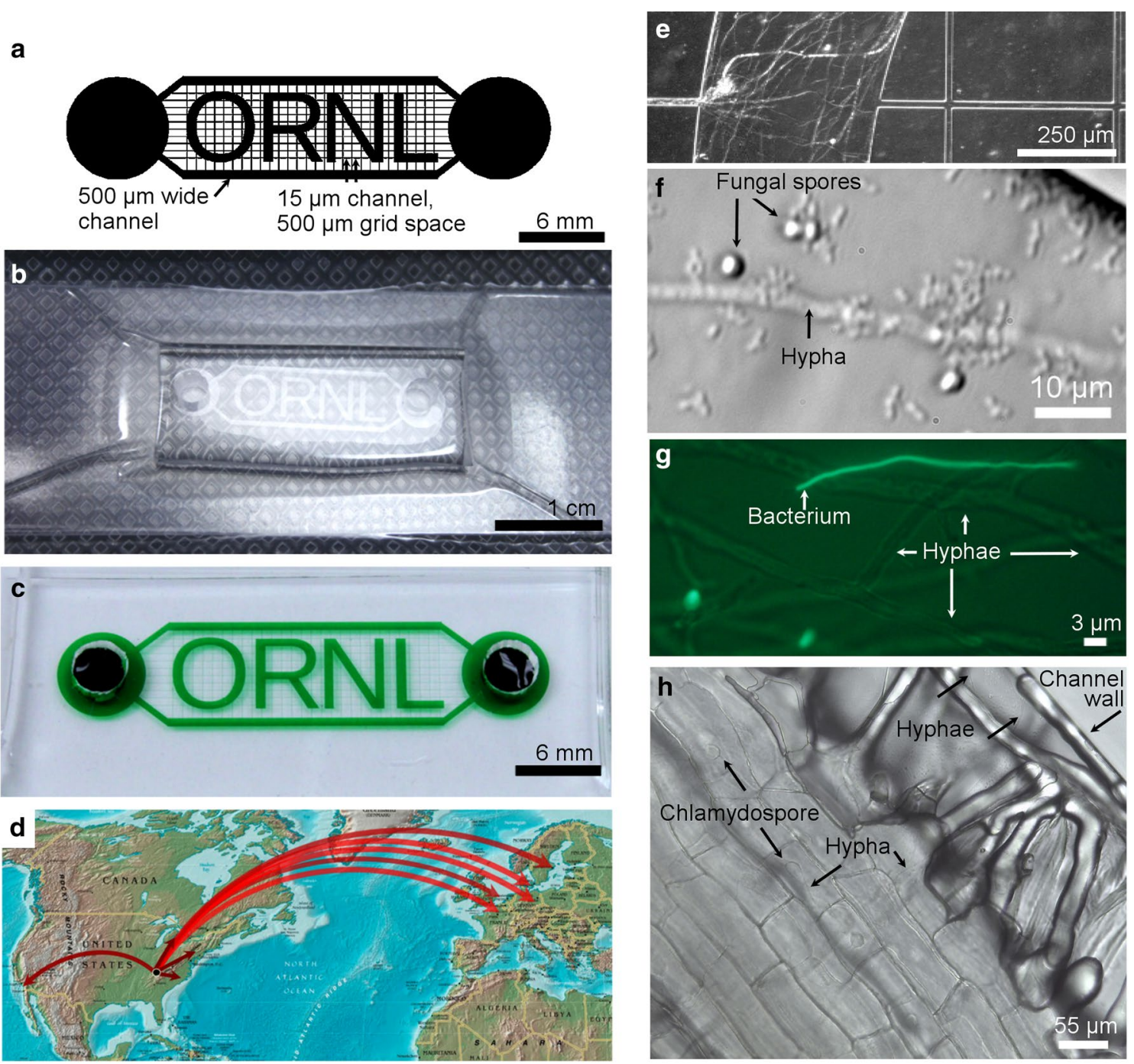

Fig. 5 Ready-to-use microfluidics for studying the biology of branched structures. a Schematic design of a four-chamber (O R N L) microfluidic architecture with two end-wells for cultures ( $6 \mathrm{~mm}$ circles). The interconnecting channel grid dimensions are shown, $15 \mu \mathrm{m}$ wide channels spaced by $500 \mu \mathrm{m}$ gap. Boundary channels framing the four chambers and grid are $500 \mu \mathrm{m}$ wide. b A sterile vacuum-packaged microfluidic chip ready for shipment, and ready to use. c An ORNL microfluidic chip with green dye in channels. d Map showing distribution of 28 ready-to-use ORNL chambers to 18 potential collaborators for fungal and other branched biology research (United States: Michigan, New York, North Carolina, and California. Europe: France, Germany, Netherlands, Denmark, Hungary, Sweden, and Switzerland0. e-h Results from implementing ready-to-use microfluidics in collaborative labs. e Rapidly growing M. elongata AG77 navigates the peripheral channels to fill the ORNL chambers of the device. $\mathbf{f}$ Microfluidic co-culture of Neurospora crassa and Pseudomonas fluorescens in microfluidic cultures (bacteria $1 \mu \mathrm{m}$, hypha $3 \mu \mathrm{m}$ ). $\mathbf{g}$ Merged image of bright field and fluorescence image of Paraburkholderia caribensis and M. elongata. $\mathbf{h}$ Nicotiana attenuata root grown in the presence of endophytic fungi, M. elongata NVP64+; fungal hypha and chlamydospores are observed within the plant root

We vacuum-packed sterile PDMS-based microfluidics for distribution to potential collaborators through a workshop on bacterial-fungal interactions. The ORNL microfluidic system is designed to contain two outer boundary channels and four central chambers in the formation of letters, "ORNL" (Fig. 5a). Upon removal of the microfluidic chamber from the vacuum-sealed pouch, the channel array is primed by adding fluid to all ports of the microfluidic channel system (Fig. 5b, c). 28 sample chambers were distributed to 18 fungal biologists within the United States (Michigan, New York, North Carolina) and throughout Europe (France, Germany, Netherlands, Denmark, Hungary, Sweden, and Switzerland) (Fig. 5d). Of the responses, at least 13 samples from 9 collaborators yielded 6 successful cultures. Figure $5 \mathrm{e}-\mathrm{h}$ demonstrates a sample of the range of results achieved from 
successful cultures in microfluidics primed after removal from the vacuum package. Of the 7 unsuccessful reports, three samples only partially filled, and three containers reportedly lost vacuum by handling, and one sample was neglected until long after the 'best use by date.' A discussion on the contributing factors competing with complete successful deployment is contained in the following discussion section.

Successful implementation of ORNL-chamber microfluidics to visualize fungal-bacterial and fungalplant interactions was achieved through collaborative exchanges (Fig. $5 \mathrm{f}-\mathrm{h}$ ). Bacterial-fungal interactions can occur between Neurospora crassa and P. fluorescens In5 (Fig. 5f). The bacterium was isolated from Greenlandic soil which is naturally suppressive to plant infections by fungal pathogens [51]. Since some antagonistic bacteria are known to attach to fungal hyphae and parasitize the fungus without penetrating the hyphae [53] it was of interest to investigate if the In 5 isolate attach to fungi. Neurospora crassa was selected for growth in the chamber for its fast growth and wide hyphae. As can be seen in Fig. $5 f$ the bacteria may attach to, or highly associate with, the fungus. Further studies are warranted to determine if $N$. crassa forms microcolonies along the growing hypha.

With chambers and boundary channels available for high-resolution imaging, the ORNL microfluidic devices were used to visualize fine-scale interactions between a plant growth promoting bacterium Paraburkholderia caribensis and Mortierella elongata NVP64+, a fungus that naturally hosts endobacteria belonging to Burkholderiaceae. We were specifically interested in whether Paraburkholderia caribensis is able to enter the mycelium of M. elongata. A co-culture of M. elongata and a GFPtransformed strain of $P$. caribensis were initiated in an ORNL chamber, each inoculated in their own port. For this microbial pair we did not see a strong interaction between partners, under these conditions at 10 days in culture; however, a number of fluorescent cells characterized by single long sinuous shape were observed (Fig. $5 \mathrm{~g}$ ) when co-cultured with $M$. elongata, in addition to the typical bacilli and diplobacilli morphologies prevalent with this bacterium. This was repeated and confirmed by fluorescence microscopy. This morphology of P. caribensis has not been observed previously, and it is not known what triggers this morphological switch in the bacterium.

To begin probing mechanisms of endophytic growth in $M$. elongata, we introduced germinated Nicotiana attenuata seeds into one end of the microfluidic wells of ORNL vacuum-packed microfluidics. The other of the two ports was inoculated with M. elongata NVP64. Within 1 week of growth we observed fungal hypha and chlamydospores, or vesicle-like structures, of $M$. elongata within the plant roots (Fig. 5h). Fungal growth within the root hairs was not observed in this sample suggesting that $M$. elongata may enter roots through epidermal cells.

To better visualize and quantify the rate of cytoplasmic streaming in M. elongata NVP64+, we used ORNL microfluidic devices. Reverse cytoplasmic streaming was observed in M. elongata NVP64+ using these microfluidic chambers, with cellular vesicles and contents moving in opposite directions (Additional file 4: Figure S3). Consistent with prior reports for cytoplasmic streaming in fungi $[67,68]$, clear cytoplasmic paths containing relatively fast velocity streaming and slow velocity streaming were observed, as evidenced by the black streaks running in straight diagonal and curved lines (Additional file 4: Figure S3).

\section{Discussion}

In this work we sought to overcome the common frustrations present with adopting microfluidic technology that limits the successful incorporation of microfluidics into traditional biology. The highlight of this work extends beyond a mere description of our approach to improving the accessibility to microfluidics, but also includes results from successful tests of microfluidics from collaborative works. First, we briefly discuss our approach to overcome these barriers including helpful tips for new users of microfluidics. In the remaining discussion we delineate the benefits and implications of ready-to-use microfluidics. We also highlight the examples and illuminate future directions of research possibilities that can be realized by microfluidics made easier.

\section{Barriers to microfluidics in biology}

Barriers to implementing microfluidics in science and research settings span a range of categories, from operational barriers (protocols and the degree of subjectmatter or device sophistication) to technical barriers (facilities and equipment) to individual barriers (experience and access to collaborators). We introduce a process that aids in removing technical and operational barriers through our design and packaging process. Nevertheless, individual experience and repetition is still required to maximize the use of microfluidic systems in biological science.

Fluidic priming is a common operational barrier to implementing microfluidics. The process of loading the microfluidic channel with fluid without introducing bubbles is a common hurdle in the implementation of microfluidics in biological labs. Filling the channels with fluid while eliminating bubbles may seem to be a trivial problem to one trained in the field, but it can be a tremendous barrier to entry for the microfluidic novice. Other technical barriers such as available equipment 
and facilities (for example, house vacuum) can put new collaborators, beginning to work with microfluidics, at a significant disadvantage or discourage collaboration. Syringe pumps are not typically available within biological labs; therefore, other means must be employed to prime the channels with fluid, or significant time and finances are a needed to invest in new pumping systems. A reliable alternative to priming microfluidics without syringe or pneumatic pumps is to equilibrate the PDMS channels in a vacuum chamber, or desiccator, then upon releasing the vacuum, immediately supply fluid to the channels (e.g. water, cell culture media, food color dye, oil, solvents) [54,69]. As the PDMS equilibrates with the local atmosphere, the material efficiently absorbs the air from the channels and is displaced by fluid from the fluid-filled ports. However, even for this simple approach, much time is spent solving this limitation on a lab-to-lab basis-regardless of distance between labs-due to the lack of vacuum supplies and equipment, and the variability in vacuum quality. House vacuum supplies, if available for degassing PDMS, are typically low vacuum $(\sim 70 \mathrm{kPa})$ and require long vacuum equilibration times to achieve enough drawing capacity to completely fill the channels with fluid. Vacuum pumps $(>80 \mathrm{kPa})$ may be available or can be purchased; however expensive equipment investments are typically unwarranted until the feasibility and promise of the microfluidic technologies have been established for collaborative investigators.

The design and architecture of a microfluidic system can potentially be a barrier to this enabling technology. While a detailed discussion on effective architectural elements for easily implementing microfluidic platforms is beyond the scope of this work, it should be noted that depending on the volume of the microfluidic architecture, house vacuum may be vastly insufficient in providing enough drawing capacity to completely prime microfluidic channels with fluid. As an alternative, inert gasses can be connected through specialized connections to push the fluid into the channels to displace bubbles through the material, here again, specialized supplies are needed.

To overcome common technical barriers that hinder new microfluidics experimentation, we sought to simplify the fluidic priming process by using a common vacuumbased approach that fills-on-demand. This inexpensive and commercially available packaging process allows for the retention of a vacuum potential within PDMS-based microfluidics for up to 2 weeks when stored under vacuum, thus preserving the ability to prime the microfluidics when returning the PDMS material to atmospheric conditions (Fig. 2). We also eliminated the need for the end-user to determine and incorporate sanitization processes for an unfamiliar technology; we pre-cleaned the microfluidics and included sanitization into the assembly, vacuum, and packaging process. We increased the accessibility to microfluidics by demonstrating a process that decreases the cost-of-investment and minimizes the steep learning curve. We disseminated microfluidics to potential collaborators for testing and use. Through our simple, inexpensive, effective, and easy-to-use process, academic labs can deliver a valuable microfluidic product for biologists to use for resolving the spatial and temporal dynamics of biological interactions (e.g. bacterial-fungal, bacterial-plant, and fungal-plant interactions).

\section{Benefits and implications}

Microfluidics are ideally suited to benefit studies of branched biological systems and mixed community interactions, for they enable physically and chemically controlled microenvironments. In nature, branched structures are capable of extending into multiple spatial domains for resource exploitation (plants and fungi), signal transduction and integration (neurons and fungi), mechanical support (plants and fungi), mass transport (plants, fungi, neurons), and cellular/organismal adaptation and regulation $[55,56]$.

The robust and inert construction of microfluidics enables pre-priming, filling, and shipping of hydrated microfluidics, with or without cultures. Preconditioning of microfluidics may be useful for initiating cultures prior to shipment, or in collecting and preserving field samples.

Incorporating agar plugs (Fig. 3b) into microfluidics substantially delays drying and provides an anchor for fungal cultures. Additionally, agar can be poured around the periphery of the microfluidics within the dish to aid in hydrating the PDMS and moistening the chamber for long-term growth and observation without fluid spillage and shearing cells.

With our approach, potential users need only open and fill the microfluidic platform to prepare samples for high-resolution imaging and can forego learning the device fabrication, assembly, sanitizing, and other preparation processes. For example, we tested our vacuum preconditioning process before deploying to collaborators, we packaged and germinated Arabidopsis seeds on chip. The channels filled with fluid, the majority of seeds germinated down the channel, and the cultures remained sterile, all without an overall impact to the root length. From these tests, we learned that the ability to vacuum seal and viably store seeds in a microfluidic platform has the potential to greatly enhance collaborations within and across disciplines. The low cost and portability of the Food Saver system also allows for field researchers to sow collected seeds or spores directly into a microfluidic platform and vacuum 
package for later use, thus preserving the seed with native microbes.

The process of preparing inoculated cultures in microfluidics saves time and eliminates initial barriers to implementing microfluidics. For example, Laccaria bicolor is a slow growing fungus and the culture can begin to be established before or during shipment. To confront this challenge, we prepared microfluidics on glass-bottomed dishes, then primed with culture media and agar, inoculated the central compartment with Laccaria fungi prior to sealing the dishes and shipping the samples to collaborators for bacterial inoculation and observation of bacterial-fungal interactions. After sustained development of Laccaria in the microfluidics during shipment and extended culture, transgenic bacteria were introduced and documented with microscopy (Fig. 3g). Biofilms formed on both bacterial-fungal co-cultures within 1 month and improve access to imaging bacterial-fungal interactions. While beneficial for some samples, this process is not suitable for all experimental conditions, cells, or organisms. Indeed, most biological samples cannot be maintained so easily during transport, (mammalian cells for example); therefore, a more ready-to-use approach is needed to expedite collaborative exchanges by overcoming initial barriers to implementing microfluidics in biology.

As an alternative to preparing cultures for transport, we propose a ready-to-use approach where microfluidics can be preconditioned dry, with vacuum, or hydrated and conditioned with agar to prevent drying and to provide an immediate-use product for the recipient to inoculate.

\section{Deployment of vacuum-packed microfluidics}

After characterizing the 'ready-to-use microfluidics', we prepared spoke-wheel microfluidics and characterized the filling time as a function of post-packaging storage time. From these samples, we determined a 2-week window for a 'best-use by' date. To deploy ready-to-usemicrofluidics with this preparation process, we fabricated an "ORNL" microfluidic architecture for testing and use by potential domestic and international collaborators. From our experience, the best process for loading chambers and successful culture is to initiate microfluidic priming within a 2-day shipping window. Longer shipments or travel may increase the time to fill, or rate of failure for this microfluidic preparation. One possible explanation for increased fill times or incomplete filling after 2 weeks in vacuum storage includes handling-induced loss of vacuum to the packaged system. Another possible explanation includes, age- and environmental-related (temperature) aging or stiffening of the elastomeric material. For example, twenty-eight sample chambers were deployed in response to interests communicated at a bacterial-fungal interactions workshop. Factors influencing the rate of successfully obtained cultures include, travel time, sample handling and storage during transit, and the learning curve for handling and filling microfluidics. Notwithstanding these possible limitations, some users reported that the devices were simple and easy to use, while others needed more replicates to successfully implement microfluidics in their experimental system. In this regard, we cannot overlook the procedural requirements of the biological system being integrated into the microfluidic chip. Culturing cells at room temperature without specialized gaseous environmental culture conditions (e.g. fungal cultures) may be easier and more forgiving, thus providing a higher apparent 'success rate' than trying to implement microfluidics with a biological protocol were time, temperature, and gas concentrations are all critical (e.g. anaerobic cultures and mammalian cell cultures). By extension, learning to use microfluidics in a biological safety cabinet is more challenging than working with them on a benchtop. Overall, we were pleased with the ability of this packaging processes to accelerate collaborative research and to quickly achieve productive results. It is our goal that this vacuum-packing process can be adopted and decrease the barriers to implementing microfluidics by providing a process to get microfluidics in-hand to end-users for testing and implementing in biological science.

\section{Focus areas and future directions}

Future research on fungal-bacterial interactions with bacteria could include fluorescent vitality stains for assessing fungi and bacteria, the use of fluorescence reporters for monitoring gene expression activity in microbes, or the use of chamber doors for sampling cells, nucleotides or metabolites from interacting tissues. Of special interest would be to investigate (1) if the architecture of the chamber can influence the outcome of a biological interaction related to previous studies, (2) how bacteria and fungi enter and grow within plant roots, (3) how environmental conditions influence cytoplasmic flow of cellular contents, or the colonization and community structure of the rhizosphere.

Microfluidic platforms have proven to be indispensable tools for probing processes of cellular function, organismal behavior, and environmental interactions. While biomedical disciplines have greatly benefited from the discoveries enabled by microfluidics [22], botany and mycology are ripe for microfluidic-enabled discoveries and solutions. Focus areas include, but are not limited to, hyphal chemotropism, fungal pathogenecity, 
tripartite interactions of the rhizosphere. More specifically, bacterial-fungal interaction studies are also in their infancy, as evidenced by much higher resolution studies [70-72]. While microfluidics offer the opportunity to engineer microcosms for probing the physical, chemical and biologicals aspects of multispecies interactions, these devices are convenient (but not required) for such imaging studies in general. Plant-fungal mutualism studies offer ample promise, and plant-bacteria interactions are valuable as well in medicine as for bioenergy, food crop production, and purifying natural resources [57-60].

Prior to deploying vacuum-equilibrated microfluidics for studying the biology of branching structures, we developed an architecture that enables low-density, high resolution access to branched hyphae for fungalbacterial interaction studies. Spoke-wheel microfluidics for ectomycorrhizal bacterial-fungal interactions were tested in-house before shipping to international colleagues for implementation (Fig. 3). The purpose of our microfluidic design was to restrict the hyphal growth region to a large fluidic area while maintaining a low microchannel ceiling $(11 \mu \mathrm{m})$ that confines the biological observations to a stationary fluidic microenvironment for imaging. This work demonstrates the ability to accelerate studies of defined microcosms within microfluidic platforms by incorporating fabrication and sterilization processes into the packing process for shipping and sharing.

\section{Conclusions}

We have characterized and deployed an affordable, readily available, and inexpensive process that solves the priming challenge, for new collaborators, by storing sterile dry vacuum-equilibrated microfluidics in a vacuum-sealed pouch for easy fluid priming, anytime, anywhere. This preparation and packaging process allow the end user to achieve fluid-primed microfluidics without the need for pumping systems. The need for fluidic priming at the fabrication source is eliminated, the shipment of fluids is avoided, and the ease of priming microfluidics is achieved. This process requires the fabrication source to have access to equipment for sterilizing (UV, plasma, autoclave), generating relatively high vacuum $(<81 \mathrm{kPa})$, and maintaining sterility during the packaging process (biosafety cabinet). The end user specifies and supplies the fluids, thereby alleviating the supplier from the need to fluid-match recipes, requirements $(\mathrm{pH})$, or conditions (prevent fluid aging or contamination).

We present a process that accelerates the adoption of microfluidics in labs lacking experience with the technique. The adaptation of challenging microtechnological methods across discipline boundaries can further accelerate the development and implementation of defined microcosms and engineered niches to better resolve fungal, bacterial, and plant interactions. By extension, microfluidics could also allow for the extraction or injection of biological contents for biochemical analyses and genome regulation studies, respectively. Breaking down scientific barriers through technological simplifications enables studies that have the power to move science forward toward resolving mechanisms of complex and pressing biological problems (e.g. microbial symbiosis or pathogen-host interactions) [14].

\section{Additional files}

Additional file 1. Supporting methods.

Additional file 2. Maturation of bacterial-fungal interactions in microfluidics.

Additional file 3. Dynamics of bacteria along the 'hyphal-highway' and cytoplasmic streaming within the mycelium.

Additional file 4. Bidirectional cytoplasmic streaming in fungal hyphae within microfluidic culture

\section{Acknowledgements}

This research was performed at Oak Ridge National Laboratory (ORNL). ORNL is managed by UT-Battelle, LLC, for the U.S. Department of Energy Under Contract No. DE-AC05-000R22725. The fabrication of the microfluidic platforms was carried out in the Nanofabrication Research Laboratory at the Center for Nanophase Materials Sciences, which is a DOE Office of Science User Facility. The views expressed are those of the authors and do not reflect the official policy or position of the Department of Energy or the U.S. Government. Collaborative contributions by Natalie Vande Pol, James Kremer, and David Rhodes are part of the Greg Bonito Group at Michigan State University.

\section{Authors' contributions}

LJM and JA designed and characterized the microfluidic preparation and packaging process, performed cultures and data analyses, and prepared manuscript. JL and JU tested and implemented microfluidics with fungal cultures and contributed to manuscript preparation. NVP, JK, DR, implemented microfluidics with fungal cultures. MLE implemented microfluidics for neuronal cultures. CMG, AD, and SO implemented microfluidics for bacterial fungal interaction studies and contributed to manuscript preparation. GB implemented microfluidics through mentoring (NVP, JK, DR) and contributed to manuscript preparation. RV, MJD, and STR supervised this work. All authors read and approved the final manuscript.

\section{Funding}

This work was supported in part by the Genomic Science Program, U.S. Department of Energy, Office of Science, Biological and Environmental Research, as part of the Plant Microbe Interfaces Scientific Focus Area (http:// pmi.ornl.gov). AD's lab was supported by the Laboratory of Excellence Advanced Research on the Biology of Tree and Forest Ecosystems (ARBRE; ANR-11-LABX 0002 01).

\section{Availability of data and materials}

The datasets used and/or analyzed during the current study are available from the corresponding author on reasonable request.

Ethics approval and consent to participate

Not applicable. 


\section{Consent for publication \\ Not applicable.}

\section{Competing interests}

The authors declare that they have no competing interests.

\begin{abstract}
Author details
1 Biosciences Division, Oak Ridge National Laboratory, PO Box 2008, MS 6445, Oak Ridge, TN 37831, USA. ${ }^{2}$ The Bredesen Center, University of TennesseeKnoxville, Knoxville, TN 37996, USA. ${ }^{3}$ The Center for Nanophase Materials Sciences, Oak Ridge National Laboratory, PO Box 2008, MS 6445, Oak Ridge, TN 37831, USA. ${ }^{4}$ Department of Biochemistry and Cellular and Molecular Biology, The University of Tennessee, Knoxville, TN 37996, USA. ${ }^{5}$ Biology Department, Duke University, Box 90338, Durham, NC 27708, USA. ${ }^{6}$ Department of Plant and Microbial Biology, University of California at Berkeley, Berkeley, CA 94703, USA. ${ }^{7}$ The Center for Neuroscience, University of California Davis, One Shields Avenue, Davis, CA 95618, USA. ${ }^{8}$ Institut national de la recherche agronomique (INRA), Centre INRA-Lorraine, 54280 Champenoux, France. ${ }^{9}$ Fujian Agricultural and Forestry University, Fuzhou City 350002, Fujian Province, China. ${ }^{10}$ Department of Plant, Soil and Microbial Sciences, Michigan State University, East Lansing, MI 48824, USA.
\end{abstract}

Received: 1 March 2019 Accepted: 15 May 2019

Published online: 10 June 2019

\section{References}

1. Devauchelle O, Petroff AP, Seybold HF, Rothman DH. Ramification of stream networks. Proc Natl Acad Sci USA. 2012;109(51):20832-6.

2. Perron JT, Richardson PW, Ferrier KL, Lapotre $M$. The root of branching river networks. Nature. 2012;492(7427):100-3.

3. Ebert U, Sentman DD. Streamers, sprites, leaders, lightning: from micro- to macroscales. J Phys D Appl Phys. 2008;41(23):230301.

4. Lu P, Werb Z. Patterning mechanisms of branched organs. Science. 2008;322(5907):1506-9.

5. Golding I, Cohen I, Ron IG, Ben-Jacob E. Adaptive branching during colonial development of lubricating bacteria. In: Fleury V, Gouyet JF, Léonetti $M$, editors. Branching in nature. Centre de Physique des Houches, vol 14 Berlin: Springer; 2001. p. 305-58.

6. Be'er A, Ariel G, Kalisman O, Helman Y, Sirota-Madi A, Zhang HP, et al. Lethal protein produced in response to competition between sibling bacterial colonies. Proc Natl Acad Sci USA. 2010;107(14):6258-63.

7. Be'er A, Smith RS, Zhang HP, Florin E-L, Payne SM, Swinney HL. Paenibacillus dendritiformis bacterial colony growth depends on surfactant but not on bacterial motion. J Bacteriol. 2009;191(18):5758-64.

8. Richards DM, Hempel AM, Flärdh K, Buttner MJ, Howard M, DePedro M, et al. Mechanistic basis of branch-site selection in filamentous bacteria. PLoS Comput Biol. 2012;8(3):e1002423.

9. Flärdh K, Richards DM, Hempel AM, Howard M, Buttner MJ. Regulation of apical growth and hyphal branching in Streptomyces. Curr Opin Microbiol. 2012;15(6):737-43

10. Wardell JN, Stocks SM, Thomas CR, Bushell ME. Decreasing the hyphal branching rate of Saccharopolyspora erythraea NRRL 2338 leads to increased resistance to breakage and increased antibiotic production. Biotechnol Bioeng. 2002;78(2):141-6.

11. Hallé F. Branching in plants. In: Fleury V, Gouyet JF, Léonetti M, editors Branching in nature. Centre de Physique des Houches, vol 14. Berlin: Springer; 2001. p. 23-40.

12. Martinuz A, Zewdu G, Ludwig N, Grundler F, Sikora RA, Schouten A. The application of Arabidopsis thaliana in studying tripartite interactions among plants, beneficial fungal endophytes and biotrophic plantparasitic nematodes. Planta. 2015;241(4):1015-25.

13. van Overbeek LS, Saikkonen K. Impact of bacterial-fungal interactions on the colonization of the endosphere. Trends Plant Sci. 2016:21(3):230-42.

14. Deveau A, Bonito G, Uehling J, Paoletti M, Becker M, Bindschedler S, et al. Bacterial-fungal interactions: ecology, mechanisms and challenges. FEMS Microbiol Rev. 2018;42(3):335-52.

15. Chklovskii DB. Synaptic connectivity and neuronal morphology. Neuron. 2004;43(5):609-17.
16. Tsai J, Grutzendler J, Duff K, Gan W-B. Fibrillar amyloid deposition leads to local synaptic abnormalities and breakage of neuronal branches. Nat Neurosci. 2004:7(11):1181-3.

17. Reinmets K, Dehkharghani A, Fuchs JS, Guasto S. Microfluidic quantification of yeast surface adhesion. ChemRxiv. 2018. https://doi. org/10.26434/chemrxiv.6446432.v1.

18. Sun Y, Tayagui A, Shearer H, Garrill A, Nock V. A microfluidic platform with integrated sensing pillars for protrusive force measurements on neurospora crassa. In: IEEE micro electro mechanical systems (MEMS). IEEE; 2018. p. 1116-9.

19. Jones S Jr, Spivey E, Rybarski J, Finkelstein I. A microfluidic device for massively parallel, whole-lifespan imaging of single fission yeast cells. Bio-protocol. 2018. https://doi.org/10.21769/BioProtoc.2783.

20. Li X, Zhang X, Liu Q, Zhao W, Liu S, Sui G. Microfluidic system for rapid detection of airborne pathogenic fungal spores. ACS Sens. 2018:3(10):2095-103.

21. Beneyton T, Thomas S, Griffiths AD, Nicaud J-M, Drevelle A, Rossignol T. Droplet-based microfluidic high-throughput screening of heterologous enzymes secreted by the yeast Yarrowia lipolytica. Microb Cell Fact. 2017:16(1):18

22. Millet LJ, Gillette MU. New perspectives on neuronal development via microfluidic environments. Trends Neurosci. 2012;35:752-61.

23. Held M, Lee AP, Edwards C, Nicolau DV. Microfluidics structures for probing the dynamic behaviour of filamentous fungi. Microelectron Eng. 2010;87(5-8):786-9.

24. Hanson KL, Nicolau DV, Filipponi L, Wang L, Lee AP, Nicolau DV. Fungi use efficient algorithms for the exploration of microfluidic networks. Small. 2006;2(10):1212-20.

25. Hu R, Li F, Lv J, He Y, Lu D, Yamada T, et al. Microfluidic analysis of pressure drop and flow behavior in hypertensive micro vessels. Biomed Microdevices. 2015;17(3):60.

26. Stanley CE, Stockli M, van Swaay D, Sabotic J, Kallio PT, Kunzler M, et al. Probing bacterial-fungal interactions at the single cell level. Integr Biol. 2014;6(10):935-45.

27. Massalha H, Korenblum E, Malitsky S, Shapiro OH, Aharoni A. Live imaging of root-bacteria interactions in a microfluidics setup. Proc Natl Acad Sci USA. 2017;114:4549-54.

28. Morrell-Falvey JL, Alexandre-Jouline GM, Sarles SA. Microscope slide-in chamber. USD754871 S1; 2016. US Patent App. 29/509,993.

29. Aufrecht JA, Timm CM, Bible A, Morrell-Falvey JL, Pelletier DA, Doktycz MJ, et al. Quantifying the spatiotemporal dynamics of plant root colonization by beneficial bacteria in a microfluidic habitat. Adv Biosyst. 2018;2(6):1800048

30. Geng T, Bredeweg EL, Szymanski CJ, Liu B, Baker SE, Orr G, et al. Compartmentalized microchannel array for high-throughput analysis of single cell polarized growth and dynamics. Sci Rep. 2015;5:16111.

31. Grossmann G, Guo W-J, Ehrhardt DW, Frommer WB, Sit RV, Quake SR, et al. The RootChip: an integrated microfluidic chip for plant science. Plant Cell. 2011:23(12):4234-40.

32. Mu X, Zheng W, Sun J, Zhang W, Jiang X. Microfluidics for manipulating cells. Small. 2013;9(1):9-21.

33. Kovarik ML, Gach PC, Ornoff DM, Wang Y, Balowski J, Farrag L, et al. Micro total analysis systems for cell biology and biochemical assays. Anal Chem. 2012;84(2):516-40.

34. Pagano G, Ventre M, lannone M, Greco F, Maffettone PL, Netti PA. Optimizing design and fabrication of microfluidic devices for cell cultures: an effective approach to control cell microenvironment in three dimensions. Biomicrofluidics. 2014;8(4):046503.

35. Kim S, Kim HJ, Jeon NL, Shanks RH, Nagiec MJ, Errede B, et al. Biological applications of microfluidic gradient devices. Integr Biol. 2010;2(11-12):584.

36. Zervantonakis IK, Hughes-Alford SK, Charest JL, Condeelis JS, Gertler FB, Kamm RD. Three-dimensional microfluidic model for tumor cell intravasation and endothelial barrier function. Proc Natl Acad Sci USA 2012;109(34):13515-20

37. Prentice-Mott HV, Meroz Y, Carlson A, Levine MA, Davidson MW, Irimia D, et al. Directional memory arises from long-lived cytoskeletal asymmetries in polarized chemotactic cells. Proc Natl Acad Sci USA. 2016;113(5):1267-72

38. Chiu DT, deMello AJ, Di Carlo D, Doyle PS, Hansen C, Maceiczyk RM, et al. Small but perfectly formed? Successes, challenges, and opportunities 
for microfluidics in the chemical and biological sciences. Chem. 2017;2(2):201-23.

39. Jain A, Graveline A, Waterhouse A, Vernet A, Flaumenhaft R, Ingber DE. A shear gradient-activated microfluidic device for automated monitoring of whole blood haemostasis and platelet function. Nat Commun. 2016;7:10176.

40. Möller FM, Kriegel F, Kieß M, Sojo V, Braun D. Steep pH gradients and directed colloid transport in a microfluidic alkaline hydrothermal pore. Angew Chem Int Ed. 2017;56(9):2340-4.

41. Schwarz J, Bierbaum V, Merrin J, Frank T, Hauschild R, Bollenbach T, et al. A microfluidic device for measuring cell migration towards substratebound and soluble chemokine gradients. Sci Rep. 2016;6:36440.

42. Millet LJ, Doktycz MJ, Retterer ST. Nanofluidic interfaces in microfluidic networks. J Vac Sci Technol B. 2015;33(6):06FM01.

43. Boyd-Moss M, Baratchi S, Di Venere M, Khoshmanesh K. Self-contained microfluidic systems: a review. Lab Chip. 2016;16:3177-92.

44. Dimov IK, Basabe-Desmonts L, Garcia-Cordero JL, Ross BM, Park Y, Ricco AJ, et al. Stand-alone self-powered integrated microfluidic blood analysis system (SIMBAS). Lab Chip. 2011;11(5):845-50.

45. Choi S. Powering point-of-care diagnostic devices. Biotechnol Adv. 2016;34(3):321-30.

46. Xu L, Lee H, Jetta D, Oh KW, Park SJ, Chung C, et al. Vacuum-driven power-free microfluidics utilizing the gas solubility or permeability of polydimethylsiloxane (PDMS). Lab Chip. 2015;15(20):3962-79.

47. Li CG, Dangol $M$, Lee $C Y$, Jang $M$, Jung $H$, Porterfield $D M$, et al. A selfpowered one-touch blood extraction system: a novel polymer-capped hollow microneedle integrated with a pre-vacuum actuator. Lab Chip. 2015;15(2):382-90.

48. Li G, Luo Y, Chen Q, Liao L, Zhao J. A "place n play" modular pump for portable microfluidic applications. Biomicrofluidics. 2012;6(1):014118.

49. Liang DY, Tentori AM, Dimov IK, Lee LP. Systematic characterization of degas-driven flow for poly(dimethylsiloxane) microfluidic devices. Biomicrofluidics. 2011;5(2):024108.

50. Aufrecht JA, Ryan JM, Hasim S, Allison DP, Nebenführ A, Doktycz MJ, Retterer ST. Imaging the root hair morphology of Arabidopsis; seedlings in a two-layer microfluidic platform. J Vis Exp. 2017. https://doi. org $/ 10.3791 / 55971$

51. Michelsen CF, Watrous J, Glaring MA, Kersten R, Koyama N, Dorrestein PC, et al. Nonribosomal peptides, key biocontrol components for Pseudomonas fluorescens In5, isolated from a Greenlandic suppressive soil. MBio. 2015;6(2):e00079.

52. Kachar B. Asymmetric illumination contrast: a method of image formation for video light microscopy. Science. 1985;227(4688):766-8.

53. Cuong ND, Nicolaisen MH, Sørensen J, Olsson S. Hyphae-colonizing Burkholderia sp:: a new source of biological control agents against sheath blight disease (Rhizoctonia solani AG1-IA) in rice. Microb Ecol. 2011;62(2):425-34.

54. Nevill JT, Mo A, Cord BJ, Palmer TD, Poo MM, Lee LP, et al. Vacuum soft lithography to direct neuronal polarization. Soft Matter. 2011;7(2):343-7.

55. Tero A, Takagi S, Saigusa T, Ito K, Bebber DP, Fricker MD, et al. Rules for biologically inspired adaptive network design. Science. 2010;327(5964):439-42.
56. Rayner ADM. The challenge of the individualistic mycelium. Mycologia. 1991;83(1):48.

57. Dhamgaye S, Murray GL, Peleg AY. The influence of bacterial interaction on the virulence of Cryptococcus neoformans. Virulence. 2015;6(7):677-8

58. Peleg AY, Hogan DA, Mylonakis E. Medically important bacterial-fungal interactions. Nat Rev Microbiol. 2010;8(5):340-9.

59. Artursson V, Finlay RD, Jansson JK. Interactions between arbuscular mycorrhizal fungi and bacteria and their potential for stimulating plant growth. Environ Microbiol. 2006:8(1):1-10

60. Bonfante P, Genre A. Mechanisms underlying beneficial plant-fungus interactions in mycorrhizal symbiosis. Nat Commun. 2010;1:48.

61. Uehling J, Gryganskyi A, Hameed K, Tschaplinski T, Misztal PK, Wu S, et al. Comparative genomics of Mortierella elongata and its bacterial endosymbiont Mycoavidus cysteinexigens. Environ Microbiol. 2017;19(8):2964-83.

62. Müller A, Volmer K, Mishra-Knyrim M, Polle A. Growing poplars for research with and without mycorrhizas. Front Plant Sci. 2013:4:332.

63. Teal TK, Lies DP, Wold BJ, Newman DK. Spatiometabolic stratification of Shewanella oneidensis biofilms. Appl Environ Microbiol. 2006;72(11):7324-30.

64. Chen Y, Stevens B, Chang J, Milbrandt J, Barres BA, Hell JW. NS21: redefined and modified supplement B27 for neuronal cultures. J Neurosci Methods. 2008;171(2):239-47.

65. Miquel Guennoc C, Rose C, Labbé J, Deveau A. Bacterial biofilm formation on the hyphae of ectomycorrhizal fungi: a widespread ability under controls? FEMS Microbiol Ecol. 2018;94(7):fiy093.

66. Labbe JL, Weston DJ, Dunkirk N, Pelletier DA, Tuskan GA. Newly identified helper bacteria stimulate ectomycorrhizal formation in Populus. Front Plant Sci. 2014;5:579.

67. Roper M, Simonin A, Hickey PC, Leeder A, Glass NL. Nuclear dynamics in a fungal chimera. Proc Natl Acad Sci USA. 2013;1 10(32):12875-80.

68. Schmieder SS, Stanley CE, Rzepiela A, van Swaay D, Sabotič J, Nørrelykke $\mathrm{SF}$, et al. Bidirectional propagation of signals and nutrients in fungal networks via specialized hyphae. Curr Biol. 2019;29(2):217-228.e4.

69. Millet LJ, Lucheon J, Standaert RF, Retterer ST, Doktycz MJ. Modular microfluidics for point-of-care protein purifications. Lab Chip. 2015;15(8):1799-811.

70. Kohlmeier S, Smits THM, Ford RM, Keel C, Harms H, Wick LY. Taking the fungal highway: mobilization of pollutant-degrading bacteria by fungi. Environ Sci Technol. 2005;39(12):4640-6.

71. Berthold T, Centler F, Hübschmann T, Remer R, Thullner M, Harms H, et al. Mycelia as a focal point for horizontal gene transfer among soil bacteria. Sci Rep. 2016;6(1):36390.

72. Worrich A, Stryhanyuk H, Musat N, König S, Banitz T, Centler F, et al. Mycelium-mediated transfer of water and nutrients stimulates bacterial activity in dry and oligotrophic environments. Nat Commun. 2017;8:15472.

\section{Publisher's Note}

Springer Nature remains neutral with regard to jurisdictional claims in published maps and institutional affiliations.
Ready to submit your research? Choose BMC and benefit from:

- fast, convenient online submission

- thorough peer review by experienced researchers in your field

- rapid publication on acceptance

- support for research data, including large and complex data types

- gold Open Access which fosters wider collaboration and increased citations

- maximum visibility for your research: over 100M website views per year

At BMC, research is always in progress.

Learn more biomedcentral.com/submissions 